STEROID RECEPTORS Allosteric Proteins?

from a Correspondent

BINDING of a steroid to specific cytoplasmic receptor proteins is thought to be the first critical step in the pathway leading to modification of gene expression in target tissues. According to all current hypotheses the steroid-receptor complexes migrate from the cytoplasm into the nucleus, but controversy exists as to whether they then bind to DNA, to non-histone proteins, or to both, or deliver the steroid to other receptor proteins. It is also argued that the receptor proteins have an additional regulatory role in the cytoplasm.

Whichever particular hypothesis is favoured, investigation of the nature and properties of the cytoplasmic receptor proteins is undoubtedly of high priority, not only for understanding how steroids work but also for clues as to how other cytoplasmic proteins may influence gene expression. Unfortunately, attempts to isolate and purify steroid receptors on a large scale have so far not met with much success, so partially purified or crude cytoplasmic extracts have been used. Nevertheless, even this approach has enabled some interesting predictions about steroid receptor behaviour to be made, the latest of which is to be found in an article in the Journal of Molecular Biology by Rousseau, Baxter and Tomkins $(67,99 ; 1972)$, who have used as a model system the induction by glucocorticoids of tyrosine aminotransferase (TAT) in hepatoma tissue culture cells.

Earlier experiments using whole cells had shown that steroids could be divided into four classes on the basis of their ability to influence TAT induction, "optimal" inducers such as corticosterone and dexamethasone stimulated a large (up to ten-fold) increase in TAT activity, whereas "sub-optimal" inducers (such as 11- $\beta$-OH-progesterone) produced only a small induction of TAT even at high concentrations, and reduced the level of induction achieved by a given amount of dexamethasone. "Antiinducers" such as progesterone and 17- $\alpha$-methyl-testosterone also inhibited induction by dexamethasone but were themselves unable to induce significant amounts of TAT. Finally, "inactive" steroids neither produced nor inhibited TAT induction.

These observations led to the proposal (Samuels and Tomkins, ibid., 52, 57 ; 1970) that the glucocorticoid receptor is an allosteric protein existing as an equilibrium mixture of two conformational states, only one of which is active in eliciting TAT induction. According to this model, optimal inducers bind to the active form of the receptor and shift the equilibrium in its favour, whereas anti-inducers bind to the inactive form and reduce the availability of active receptor. Sub-optimal inducers can bind to both forms of the protein, whereas inactive steroids do not bind at all.

Rousseau et al. have now taken these experiments a step further and, using a simple and rapid assay, have looked at the ability of different steroids to bind in vitro to cytoplasmic extracts of hepatoma cells. They have found that optimal inducers bind to a single class of receptor sites with an affinity proportional to their potency in inducing TAT in whole cells. The anti-inducer progesterone also binds to a single class of receptor sites and competitively inhibits dexamethasone binding. Assuming that the receptors for progesterone and dexamethasone are the same (and the number of binding sites calculated for both steroids is very close), the extent to which the anti-inducer competes is predictable from the relative affinities of the two steroids for their binding sites, and accounts nicely for the ability of progesterone to inhibit TAT induction by dexamethasone in whole cells. Thus the behaviour of the different steroids in cytoplasmic extracts closely parallels their biological activity and is still compatible with the model predicted by Samuels and Tomkins. Rousseau et al. go on to describe experiments designed specifically to test this model.

It had been noted previously that un- complexed receptors (presumably in the inactive form) were more thermolabile (and also more tightly bound to glass and other surfaces) than those complexed with dexamethasone. It was argued that if progesterone bound to the inactive form of the receptor, the progesterone-receptor complex should be more thermolabile than the dexamethasone or cortisol-bound (active) receptor, and this is indeed what is observed. In addition the different kinetics of the association of dexamethasone, cortisol and progesterone with the receptor are consistent with a conformational change taking place after binding of the inducers, but not the anti-inducer. Another piece of evidence in favour of the allosteric model is the observation that, unlike the dexamethasone-bound form of the receptor, the progesteronebound molecules do not accumulate in the cell nucleus.

By modifying different groups on the progesterone and corticosterone molecules, Rousseau et al. were able to establish which parts of the steroid structure determine the affinity of the compound for the active or inactive conformations of the receptor. This sort of approach could obviously be very useful in the design of new steroid drugs, but it remains to be seen whether natural anti-inducers play any part in modulating the activity of glucocorticoids in vivo.

\title{
Model for Type I Supernovae
}

MANY explanations have been put forward to account for Type I supernovae, but none has yet proved entirely satisfactory. The latest step in the slow progress towards an understanding of these violent eruptions comes from F. D. A. Hartwick, who suggests (in next Monday's Nature Physical Science, June 26) that explosive hydrogen burning caused by mass transfer in a binary system could be the driving force.

The particular difficulty in constructing models of Type I supernovae is that they occur chiefly in elliptical galaxies which are thought to contain stars with masses $\lesssim 1 M_{\odot}$. It is not too difficult to explain how a very massive star might explode as a result of a sudden collapse after one stage in the nuclear burning process has been exhausted. In that case, collapse and consequent heating of the stellar interior can occur until the next fusion reaction begins, and this can certainly take place explosively. But a low mass star should, in theory, sit quietly, burning its nuclear fuel as far as possible and then cooling into a white dwarf.

In contrast to this naive picture, Hartwick's models produce violent Type I supernovae in systems where one component is very low mass indeed-about $0.1 M_{\odot}$. The key to the process is that such a star will evolve over a time scale of $\sim 1.23 \times 10^{9}$ yr until the central temperature is falling and the centre of the star is becoming degenerate while unburnt hydrogen remains in the outer layers-just the picture of a quiet white dwarfish old age outlined earlier. But because Hartwick's models are members of binary systems they are not left in peace to enjoy a respectable retirement. If the companion to such a $0.1 M_{0}$ star is itself about 0.7 to $1.0 M_{\odot}$, by the time its small companion is settling down the larger star will be expanding, during the normal course of evolution, and transferring mass to its small, degenerate partner. This is literally an explosive situation. A cooling, but still hot, degenerate star is being compressed by the sudden addition of mass from outside.

According to Hartwick, this could raise its internal temperature abruptly, suddenly igniting another round of nuclear burning throughout the remaining hydrogen, and resulting in a violent explosion; this can occur for only a moderate amount of mass transfer, because of the low initial mass of the smaller partner in the binary pair. More detailed calculations are clearly needed, but as it stands this model is as good as most of the other explanations of Type I supernovae proposed so far. 